\title{
«Entscheidend war die Begeisterung füir das Fach»
}

\author{
En décembre dernier, Felix Largiadèr, un des éminents chirurgiens helvétiques du \\ $\mathrm{XX}$ siècle, célébrait son $80^{\mathrm{e}}$ anniversaire. A cette occasion, celui qui a été longtemps à \\ la tête du service de chirurgie de l'hôpital universitaire de Zurich s'est offert lui-même \\ un beau cadeau: la publication de son livre «Transplantation von Organen» dans lequel \\ il porte un regard aussi complet que captivant sur sa carrière professionnelle et sur un \\ domaine de la médecine qu'il a marqué de son empreinte pendant plusieurs décennies.
}

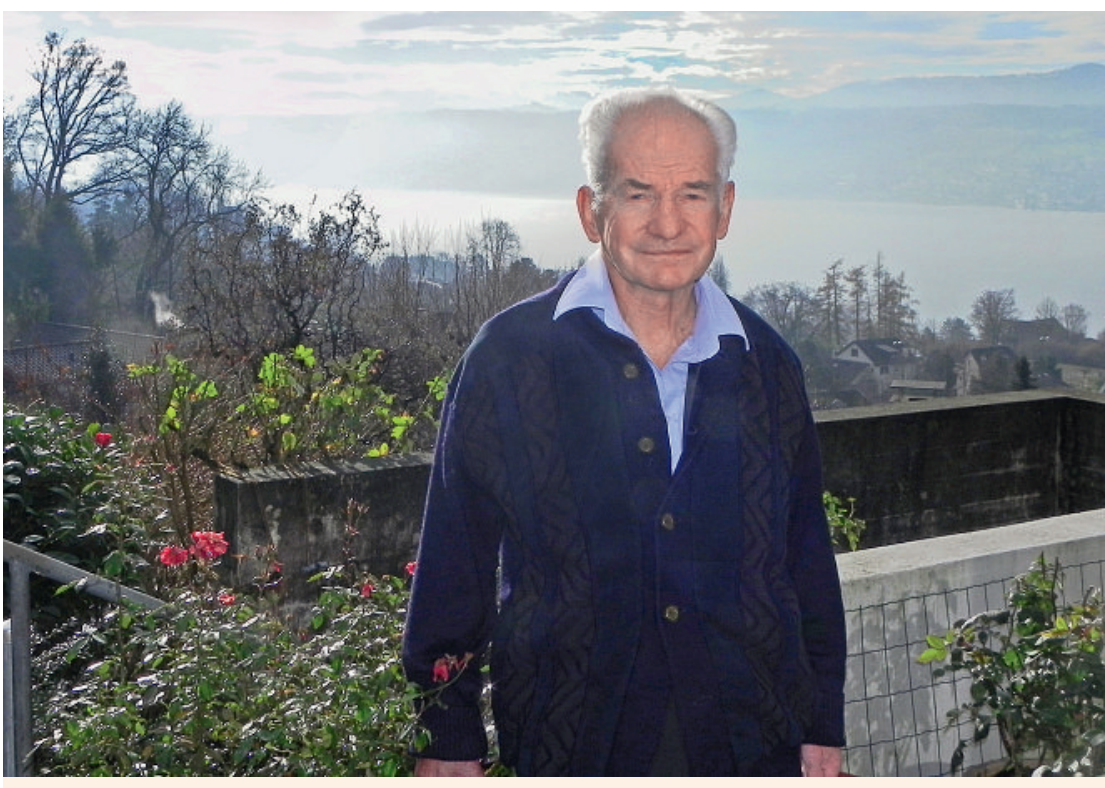

«Dass ich etwas anderes mit gleichem Einsatz gemacht hätte, kann ich mir nicht vorstellen»: der Chirurg Felix Largiadèr auf der Terrasse seines Hauses in Erlenbach.
Bruno Kesseli

bkesseli@emh.ch
Auch im Alter von 80 Jahren strahlt der eher zierliche Mann noch eine beeindruckende Energie aus. Sie leuchtet aus den wachen, im Gespräch intensiv auf das Gegenüber konzentrierten Augen, schwingt in der leisen, aber eindringlichen Stimme mit und ist im festen Händedruck spürbar. Felix Largiadèr hat es sich nicht nehmen lassen, den schreibenden Gast persönlich am Bahnhof abzuholen. Zügig meistert er am Steuer die steilen Kurven zu seinem Haus über dem Zürichsee, zieht dann wie an der Schnur gezogen im Rückwärtsgang die Einfahrt hoch und setzt den Wagen punktgenau auf die dafür vorgesehene Abstellfläche. In diesem Tempo und mit dieser Präzision wird er sein Skalpell geführt haben, denkt man sich einigermassen beeindruckt.

Seit 1967 lebt Felix Largiadèr mit seiner Frau Helene in Erlenbach an der Zürcher Goldküste, seit 1974 im eigenen Haus - «damals war ein solches Haus noch erschwinglich»-, hier wuchsen ihre fünf Kinder auf, die längst ausgeflogen sind. Herumtollende Dreikäsehochs sind im Haus dennoch keine Seltenheit, was daran liegt, dass die Familie mittlerweile um zehn Enkel angewachsen ist. Zur grossen Freude des Grossvaters: «Ich geniesse das Familienleben und empfinde es als Privileg, dass ich dafür viel mehr Zeit habe als früher.»

\section{Die Berge als Konstante}

Von der Gartenterrasse schweift der Blick über den Zürichsee bis zur Alpenkette mit ihren schneebedeckten Gipfeln. Wie die Chirurgie bilden auch die Berge eine Konstante im Leben des Felix Largiadèr. Sie haben ihn zeitlebens unwiderstehlich angezogen, wozu verschiedene Faktoren beigetragen haben mögen: Die Largiadèrs stammen ursprünglich aus dem Val Müstair, das als eines der schönsten Bergtäler der Schweiz gilt, und auch Felix Largiadèrs Mutter Gertrud Stüdli hat ihre Liebe zu den Bergen bereits vor der Hochzeit in einem Brief an den Bräutigam eindrücklich festgehalten.

Geboren und aufgewachsen ist Felix Largiadèr allerdings nicht in einer Bergregion, sondern in der Ostschweizer Gemeinde Flawil, wo sein Vater als Chefarzt des örtlichen Spitals tätig war. «Warum ich vom Bergsteigen so fasziniert bin, lässt sich letztlich nicht rational erklären: Das bin einfach ich!», setzt er müssigen Spekulationen nach dem Ursprung seiner Leidenschaft ein Ende, ganz ähnlich, wie dies herausragende Alpinisten vergangener Tage auf vergleichbare Fragen getan haben. 


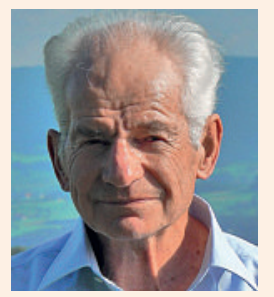

Foto:

Helene Largiadèr

\section{Felix Largiadèr}

Felix Anton Largiadèr wurde am 18. Dezember 1930 in Flawil (SG) geboren, wo er auch aufwuchs. Nach der Matura (Typ A) an der Kantonsschule St. Gallen studierte er Medizin in Genf, München und Zürich, machte 1956 sein Staatsexamen und promovierte 1959 in Zürich zum Dr. med. Seine Assistenzzeit absolvierte er in Wattwil, St. Gallen (Pathologie und ORL) sowie am Universitätsspital Zürich (Chirurgische Klinik A). 1963-65 war er Research Fellow im Departement of Surgery der University of Minnesota Hospitals in Minneapolis, USA, wo er im Rahmen eines Nachdiplomstudiums den Abschluss eines «Master of Science in Surgery» machte.

Ab 1965 wirkte Largiadèr im Universitätsspital Zürich; dort erfolgte 1967 auch die Habilitation. 1975 wurde er zum Extraordinarius, 1980 zum Ordinarius ad personam für Organtransplantationschirurgie ernannt. Von 1985 bis zur Emeritierung 1998 war er ordentlicher Professor für Chirurgie an der Universität Zürich, Vorsteher des neugeschaffenen Departements Chirurgie und Direktor der Klinik für Viszeralchirurgie im Universitätsspital Zürich. Largiadèr war 1985 Mitbegründer der Stiftung Swisstransplant, die er 1998/99 präsidierte.

\section{In Bergsteigermontur und unrasiert vor der Nachfolgekommission}

Largiadèrs Begeisterung für die Berge wird von seiner Frau Helene geteilt, und so erstaunt es nicht, dass die Familie einen Grossteil der Ferien dem Gebirgswandern und Klettern widmete. Selbst in Zeiten «fakultätspolitischer Hochspannung», während des Auswahlverfahrens für die Nachfolge des damaligen Direktors der Chirurgischen Klinik A in Zürich, Ảke Senning, als sich massgebliche Kräfte gegen eine Berufung Largiadèrs starkmachten, liess sich dieser nicht von seiner Leidenschaft abbringen. Als er kurzfristig ein Aufgebot zur Vorstellung seines Konzepts für die Neuorganisation der Chirurgie am Universitätsspital erhielt, reiste er direkt von einer Hoch-

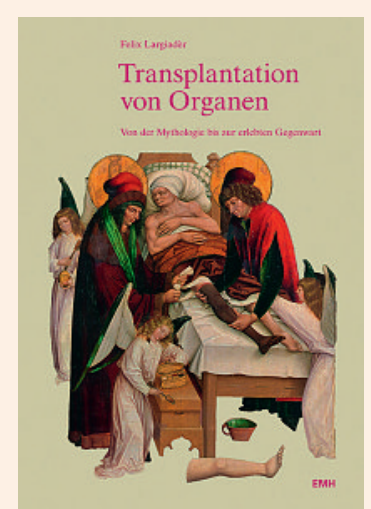

Felix Largiadèr

Transplantation von Organen

Von der Mythologie bis zur erlebten Gegenwart.

Basel: EMH Schweizerischer Ärzteverlag; 2010.

375 Seiten, 33 Abbildungen. Gebunden.

ISBN 978-3-03754-051-0

\section{"Transplantation von Organen"}

Felix Largiadèr, als Begründer und Hauptautor der «Checkliste Chirurgie» (weit über 100000 abgesetzte Exemplare) Generationen von Ärzten ein Begriff, zählt unzweifelhaft zu den Pionieren der Organtransplantation.

Ab den 60er Jahren des letzten Jahrhunderts hat er deren Entwicklung nicht nur miterlebt, sondern auch an vorderster Front mitgeprägt.

Schon als junger Assistent, «noch bevor ich selber meine erste Transplantation am Menschen gemacht hatte», schrieb er ein Buch über dieses Thema (Organ-Transplantation, Thieme Verlag 1966, nur noch antiquarisch erhältlich). Es war das erste seiner Art im deutschsprachigen Raum, ein Attribut, das auch auf sein jüngstes Werk «Transplantation von Organen» zutrifft: «Eine umfassende Darstellung der Transplantationsmedizin von der Mythologie bis in die Gegenwart gab es bisher in deutscher Sprache nicht», so der Autor. gebirgstour an und hielt seine Ausführungen vor der Nachfolgekommission in Bergsteigermontur und unrasiert - um Mitternacht war er bereits wieder auf der Grimsel, um seine Tour am Folgetag fortsetzen zu können.

Der «ungebührliche» Auftritt wurde zwar kritisiert, konnte den durchschlagenden Erfolg aber nicht verhindern: Auf den 16. April 1985 wurde Felix Largiadèr vom Zürcher Regierungsrat zum etatmässigen Ordinarius für Chirurgie, zum Vorsteher des neugeschaffenen Departements Chirurgie und zum Direktor der Klinik für Viszeralchirurgie des Universitätsspitals Zürich gewählt, und er hatte diese Ämter bis zu seiner Emeritierung im April 1998 inne.

Mittlerweile lassen es die Largiadèrs in Sachen Berg- und Klettertouren zwar ruhiger angehen, aber das ist relativ zu verstehen. Seit Felix Largiadèrs Pensionierung ist das Ehepaar beispielsweise jeden Sommer von Erlenbach ins Münstertal gewandert, auf dessen Sonnenseite sie in 2000 Metern Höhe ein «Refugium» besitzen. 13 Mal haben sie diese Wanderung schon gemacht und dabei den Alpenhauptkamm stets an einer anderen Stelle überquert.

Nicht nur die erhabenen Seiten der Bergwelt, auch ihre Gefahren hat Felix Largiadèr am eigenen Leib erlebt - mit bleibenden Folgen. Im Juli 1996 stürzte er am Mont Blanc ab und zog sich dabei ein Schädel-Hirn-Trauma zu, das seine sprachliche Ausdrucksfähigkeit nachhaltig beeinträchtigt hat. Obschon er sich nach wie vor klar und differenziert ausdrückt, ist «meine Sprache nicht mehr so schnell, verständlich und präzise wie früher». Diese plötzliche Einschränkung wird nicht einfach $\mathrm{zu}$ akzeptieren gewesen sein für einen Mann wie ihn, der an sich selbst höchste Ansprüche stellt. «Selber schuld, warum braucht er in seinem Alter noch auf so hohe Berge zu

Neben der detaillierten, dank einer Vielzahl direkter Bezüge stets lebendig-anschaulichen Schilderung der Entwicklung eines hochspezialisierten Bereichs der Medizin bietet das Buch auch einen faszinierenden Einblick in das Leben des Menschen und Chirurgen Felix Largiadèr, ohne je zur persönlichen Nabelschau zu werden.

Fortschritte, Hindernisse, Durchbrüche und Rückschläge im Bereich der Organtransplantation auf lokaler, nationaler und internationaler Ebene werden präzis und für ein breites Publikum verständlich nachgezeichnet.

Dass es dem Autor gelingt, mit lakonischer Distanz und einem Schuss trockenen Humors die (gesundheits-)politische und institutionelle Begleitmusik einer grossen Medizinerkarriere zu vermitteln, macht sein Werk auch über die rein fachlichen Aspekte hinaus zu einer reizvollen Lektüre. 
steigen, haben damals viele liebe Kollegen gesagt oder gedacht.»

\section{«Wenn eine Operation gemacht ist, ist sie gemacht"}

Mit dem Schicksal zu hadern, scheint indes nicht Largiadèrs Art zu sein. Was ihn als Bergsteiger auf Hunderte von Gipfeln gebracht hat, dürfte auch seine chirurgische Laufbahn massgeblich geprägt haben: die Fähigkeit, ein als lohnenswert erachtetes Ziel entschlossen, beharrlich und mit kühlem Kopf zu verfolgen, Risiken nicht zu scheuen, aber nie leichtfertig mit ihnen umzugehen. Seine beruflichen Ziele steckte er schon in jungen Jahren höher als die meisten seiner Weggefährten. Bereits während des Medizinstudiums erlaubte er sich den kühnen Traum, eines Tages die gesamte Chirurgie am Universitätsspital Zürich zu leiten. Dass Ehrgeiz in seiner Karriere eine nicht unwesentliche Rolle gespielt hat, räumt er ohne weiteres ein, um betont aber: «Entscheidend war die Begeisterung für das Fach.»

Das Streben an die Spitze hat für ihn zwei Seiten: «Dahinter steht sicher der Wunsch, fachlich keine höhere Instanz mehr über sich zu haben, selbst entscheiden zu können, aber auch die Bereitschaft, die Verantwortung für das eigene Tun zu tragen und sie nicht auf andere abzuschieben.» Verantwortung übernehmen, Entscheidungen fällen - «diese Art von Medizin entspricht offenbar meinem Naturell», stellt Largiadèr fest. «Wenn eine Operation gemacht ist, ist sie gemacht, und deshalb ist es eine grosse und faszinierende Aufgabe, die Operationsindikation richtig zu stellen.»

War die Chirurgie für ihn eine Berufung, oder hätte er seine Qualitäten auch in einem anderen Umfeld, etwa einer Führungsposition in der Wirtschaft, ausleben können? «Dass ich etwas anderes mit gleichem Einsatz gemacht hätte, kann ich mir nicht vorstellen», sagt Largiadèr dazu. Und er erwähnt wichtige Vorbilder, die seinen Berufswunsch geformt haben, allen voran seinen Vater, aber auch seine klinischen Lehrer im Studium, insbesondere Professor Alfred Brunner, dessen Vorlesungen ihn stark beeindruckten. «Weil mich die Chirurgie fasziniert hat, habe ich ihr fast unbeschränkt Zeit und Energie geopfert.»

\section{«Jeder Arzt muss sich gelegentlich sagen: (Das hätte ich anders machen sollen.»"}

Viel erwartete er auch von seinen Mitarbeiterinnen und Mitarbeitern, «wenn auch nicht so viel wie von mir selbst». Dass eine solche Berufsauffassung heute nicht mehr dem Mainstream entspricht, ist Largiadèr klar. Er gehört aber keineswegs zu den Vertretern seines Fachs, die den gegenwärtigen Tendenzen in der Medizin nichts Positives abgewinnen können. Vielmehr vermittelt er im Gespräch den Eindruck eines Realisten, der überzeugt ist, dass gute Ärzte auch unter den veränderten Bedingungen gute Arbeit leisten können. «Der Arzt funktioniert heute im Ver- gleich zu früher viel weniger allein, sondern als Teil der «Gesamtmedizin`. Das ist eine Entwicklung, die man nicht als gut oder schlecht bewerten kann - sie ist einfach normal.»

Nicht verändert haben sich die Ansprüche, die Largiadèr an einen guten Arzt, eine gute Ärztin stellt. «Ein guter Arzt hat eine exzellente Aus- und Weiterbildung und hält sich fachlich stets à jour. Er nimmt sich Zeit für seine Patienten, um deren Beschwerden und Anliegen genau zu erfassen und danach aufgrund seines Wissens und seiner Fähigkeiten die richtige Behandlung durchzuführen.» Als unfehlbar sieht Largiadèr sich und seinesgleichen keineswegs: «Jeder Arzt muss sich gelegentlich sagen: \Das hätte ich anders machen sollen.»»

\section{Familie vor «gesellschaftlichem Leben»}

Und die Familie? Immerhin waren da noch seine Frau und die fünf Kinder - liessen sich Familienleben und Beruf bei seinem Pensum in Klinik, Lehre und Forschung überhaupt unter einen Hut bringen? «Ich

\section{Das Geburtstagssymposium}

«Eine der Hauptaufgaben des chirurgischen Chefs war und ist es, unter seinen vielen Schülern diejenigen herauszufiltern und zu fördern, bei denen man die Fähigkeiten und den nötigen Willen spürt, gute Chirurgen zu werden.» Gemessen an der Zahl bedeutender Schweizer Chirurgen, die bei ihm «in die Schule gegangen» sind, scheint Felix Largiadèr diesen selbstgestellten Anspruch bestens erfüllt zu haben.

Einer von ihnen, Walter Weder, hat am 17. Dezember 2010 im UniversitätsSpital Zürich unter dem stimmigen Titel «Eine Höhenwanderung durch die Spezialisierung der Chirurgie» ein Symposium zum 80. Geburtstag seines Lehrers organisiert - weitere wie Daniel Candinas und Marco Decurtins waren als Redner und Gratulanten anwesend.

Die Liste der Referenten wurde komplettiert durch den Dekan der Medizinischen Fakultät der Universität Zürich, Klaus Grätz, seine Professorenkollegen Gregor Zünd, Pierre A. Clavien, Volkmar Falk und Konrad Bloch sowie die Vorsitzende der Spitaldirektion des USZ, Rita Ziegler. Felix Largiadèrs Stimme verlor ihre Festigkeit beim Schlusswort zwar nicht. Der Jubilar zeigte sich aber angesichts der Wertschätzung, die er wie auch seine Frau Helene von allen Seiten spüren durften, sichtlich gerührt. Der abschliessende Apéro, der in familiärer Atmosphäre stattfand, bot schliesslich reichlich Gelegenheit, Erinnerungen wiederaufleben zu lassen, sich fachlich und privat auf den neuesten Stand zu bringen, Zukunftsperspektiven zu entwickeln und auf den ehemaligen Chef, Weggefährten, Freund und Vater anzustossen. 


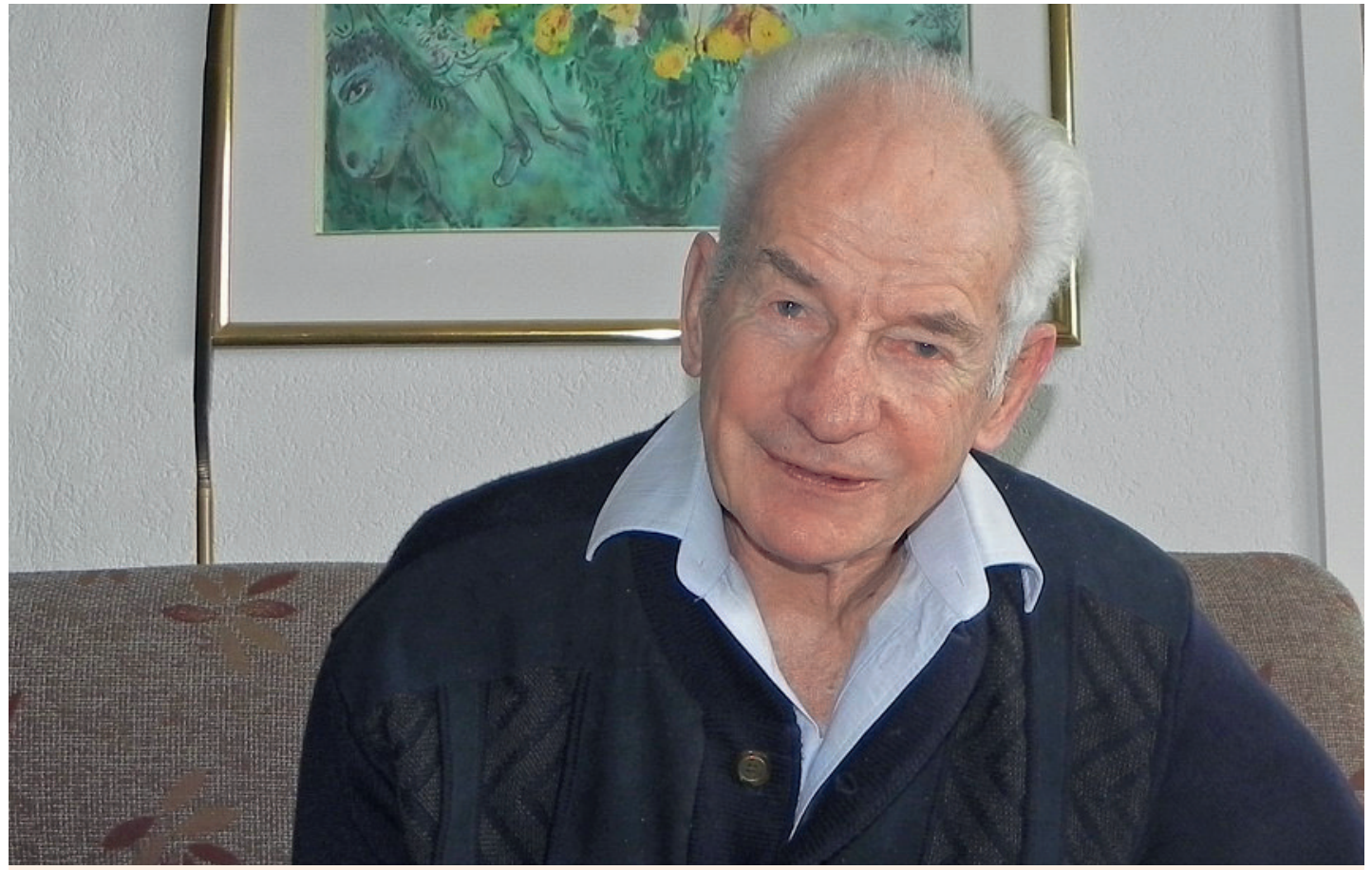

«Immer, wenn ich Lust hatte, setzte ich mich hin und schrieb»: Felix Largiadèr zur Entstehung seines Buchs «Transplantation von Organen». Foto: $B K$.

meine, dass mir dies ganz gut gelungen ist, allerdings nur, weil ich auf das (gesellschaftliche Leben` fast vollständig verzichtet habe. Dies ermöglichte mir, im Familienalltag präsent zu sein, mich beispielsweise um schulische Fragen zu kümmern und alle Ferien mit meinen Kindern zu verbringen.»

Largiadèr betont aber, dass seine Frau im familiären Bereich viel mehr geleistet habe als er, indem sie die Berufstätigkeit vorübergehend zugunsten der Kinder zurückstellte. Und nicht nur das - auch in seiner beruflichen Laufbahn habe er ihre Unterstützung als unverzichtbar erlebt: «Es war entscheidend, dass meine Frau mir beistand und alles mitmachte, was mein Beruf mit sich brachte. Ohne sie wäre meine Karriere in dieser Form nicht denkbar gewesen.»

\section{Publizistische Aktivitäten nach Emeritierung}

Obwohl der Ausspruch «Die Chirurgie war mein Leben» in seinem Fall nicht unberechtigt wäre, blieb die Sinnkrise aus, als sich Largiadèr 1998 aus dem Berufsleben zurückzog. Er widmete sich unter anderem publizistischen Aktivitäten, zeichnete als Redaktor für das «Bulletin», die offizielle Zeitschrift von Swisstransplant, verantwortlich. Die Option «Privatklinik» stand für ihn dagegen nie zur Diskussion. «Als im 68. Altersjahr stehender Chirurg sollte man wirklich einsehen, dass die operative Tätigkeit trotz ungebrochener fachlicher Faszination für die Chirurgie einmal ein Ende finden muss», schreibt er dazu im
Vorwort seines Buchs «Transplantation von Organen». Die Arbeit an diesem Werk stand in den letzten Jahren im Vordergrund, «ich fühlte mich aber nie gedrängt - immer, wenn ich Lust hatte, setzte ich mich hin und schrieb».

Und wie sehen die Perspektiven jetzt aus, da das Buch abgeschlossen und veröffentlicht ist? «Ja», meint Largiadèr mit verschmitztem Lächeln, «jetzt habe ich schon das Problem, was ich mit all der freien Zeit mache.» Um gleich hinzuzufügen: «Ich fühle mich durch Frau, Kinder und Enkel gut aufgehoben und versorgt, erfreue mich an Wandern, Bergsteigen und einem intensiven Kulturleben mit Oper, Konzert und Schauspiel - und jetzt habe ich viel zu viel geschwatzt.»

\section{Epilog}

Am Folgetag des Besuchs folgte auf elektronischem Weg eine kurze Notiz: «Gestern traf am späteren Nachmittag ein Mail des Thieme-Verlags in Stuttgart ein, wegen des anhaltenden guten Absatzes der 9. Auflage sei die Arbeit an der 10. Auflage der Checkliste Chirurgie, smeiner Checkliste, an die Hand zu nehmen. Folge: genügend Arbeit für mehr als ein Jahr, auch wenn ich das rein Fachliche an jüngere, in der Chirurgie noch aktive Mitarbeiter delegieren werde!»

So schnell ändern sich die Perspektiven. 